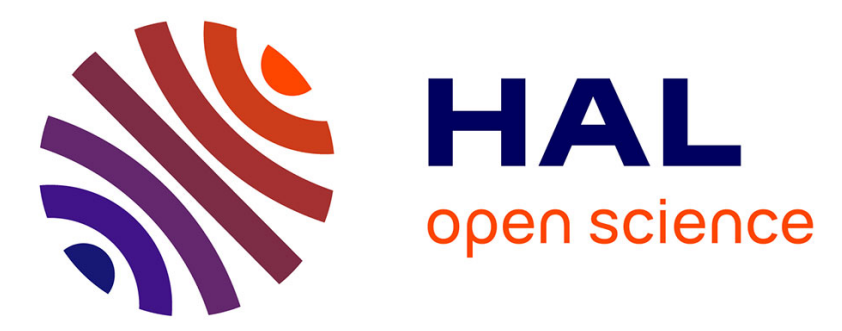

\title{
Hyperfine fields and anisotropy of the orbital moment in epitaxial Mn5Ge3 films studied by Mn55 NMR
}

\author{
R. Kalvig, E. Jedryka, M. Wojcik, G. Allodi, R. de Renzi, Matthieu Petit, \\ Lisa Michez
}

\section{- To cite this version:}

R. Kalvig, E. Jedryka, M. Wojcik, G. Allodi, R. de Renzi, et al.. Hyperfine fields and anisotropy of the orbital moment in epitaxial Mn5Ge3 films studied by Mn55 NMR. Physical Review B: Condensed Matter and Materials Physics (1998-2015), 2018, 97 (17), pp.174428 - 174428. 10.1103/PhysRevB.97.174428 . hal-01803350

\section{HAL Id: hal-01803350 \\ https://hal-amu.archives-ouvertes.fr/hal-01803350}

Submitted on 30 May 2018

HAL is a multi-disciplinary open access archive for the deposit and dissemination of scientific research documents, whether they are published or not. The documents may come from teaching and research institutions in France or abroad, or from public or private research centers.
L'archive ouverte pluridisciplinaire HAL, est destinée au dépôt et à la diffusion de documents scientifiques de niveau recherche, publiés ou non, émanant des établissements d'enseignement et de recherche français ou étrangers, des laboratoires publics ou privés. 


\title{
Hyperfine fields and anisotropy of the orbital moment in epitaxial $\mathrm{Mn}_{5} \mathrm{Ge}_{3}$ films studied by ${ }^{55}$ Mn NMR
}

\author{
R. Kalvig, E. Jedryka, ${ }^{*}$ and M. Wojcik \\ Institute of Physics, Polish Academy of Sciences, Aleja Lotników 32/46, PL-02668 Warsaw, Poland \\ G. Allodi and R. De Renzi \\ Dipartimento di Scienze Matematiche, Fisiche e Informatiche, Universitá degli Studi di Parma, I-43124 Parma, Italy \\ M. Petit and L. Michez \\ Aix Marseille Univ, CNRS, CINAM, F-13288 Marseille, France
}

(Received 19 March 2018; published 29 May 2018)

\begin{abstract}
${ }^{55} \mathrm{Mn}$ NMR was used to perform the atomic-scale study of the anisotropic properties of $\mathrm{Mn}_{5} \mathrm{Ge}_{3} / \mathrm{Ge}(111)$ epitaxial films with thicknesses between 9 and $300 \mathrm{~nm}$. The NMR spectra have been recorded as a function of strong external magnetic field applied in the film plane and perpendicular to it. Two ${ }^{55} \mathrm{Mn}$ NMR resonances have been observed, corresponding to the two manganese sites $4 d$ and $6 g$, in the hexagonal $\mathrm{D} 88$ structure; in zero field their frequency is centered around 207.5 and $428 \mathrm{MHz}$, respectively. The anisotropy of ${ }^{55} \mathrm{Mn}$ hyperfine fields between the hexagonal $c$ direction and the $c$ plane at both Mn sites was evidenced and attributed to the anisotropic term due to the unquenched Mn orbital momentum. The anisotropy of the orbital contribution to hyperfine fields was determined as $1.52 \mathrm{~T}$ in the $4 d$ site and up to $2.77 \mathrm{~T}$ in the $6 g$ site. The $4 d$ site reveals a quadrupolar interaction due to the strong electric field gradient: $V_{z z}=5.3 \times 10^{19} \mathrm{~V} / \mathrm{m}^{2}$ in this site, which is shown to be oriented along the hexagonal $c$ axis.
\end{abstract}

DOI: 10.1103/PhysRevB.97.174428

\section{INTRODUCTION}

The intermetallic $\mathrm{Mn}_{5} \mathrm{Ge}_{3}$ compound attracted a lot of research interest as a new spintronic material, a potential source of polarized carriers into Ge. Prospective applications eventuate from the high-spin polarization of the conduction electrons $(42 \%)$ [1], compatibility with conventional $\mathrm{Si} / \mathrm{Ge}$ electronics, and a Curie temperature of $296 \mathrm{~K}$, that can be further increased up to $445 \mathrm{~K}$ by adding small amount of carbon [2]. Moreover, this compound displays strong magnetocrystalline anisotropy giving a possibility to combine spintronic and magnetic recording applications [3]. $\mathrm{Mn}_{5} \mathrm{Ge}_{3}$ crystallizes in the hexagonal $\mathrm{D} 8_{8}$ structure (space group $P 6_{3} / \mathrm{mcm}$ ). The unit cell contains two formula units, with $\mathrm{Mn}$ atoms located in two crystallographic positions [4], and is schematically presented in Fig. 1. Four manganese ions located at the $4 d$ site (further denoted as $\mathrm{Mn}_{I}$ ) are surrounded at the distance of $2.54 \AA$ by six Ge ions in the first-neighbor shell. Six $\mathrm{Mn}$ ions located on $6 \mathrm{~g}$ sites (denoted as $\mathrm{Mn}_{\text {II }}$ sites) form a second-neighbor shell distant at $3.06 \AA$.

It has been shown that this compound can be successfully grown by solid phase epitaxy (SPE), consisting in a roomtemperature Mn deposition on the $\mathrm{Ge}(111)$ substrate followed by thermal annealing. Good quality epitaxial films can be obtained with (0001) hexagonal $c$ axis parallel to the Ge(111) direction [6]. A detailed study of magnetization reversal process in thin films of $\mathrm{Mn}_{5} \mathrm{Ge}_{3}$ based on the analysis of hysteresis

*Corresponding author: jedry@ifpan.edu.pl loops, revealed that in the thickness range between 10 and $20 \mathrm{~nm}$, magnetization direction at low temperatures undergoes reorientation from the in-plane to out-of-plane direction (i.e., along the hexagonal $c$ axis) and at $25 \mathrm{~nm}$ a clear signature of a multidomain structure with perpendicular orientation was observed [7]. The reorientation of magnetization direction in the thickness range 10-20 $\mathrm{nm}$ has been independently confirmed by the ferromagnetic resonance (FMR) studies in a series of $\mathrm{Mn}_{5} \mathrm{Ge}_{3}$ thin epitaxial films [8]. However, hysteresis loops as well as FMR studies provide information on the bulk magnetization and do not elucidate microscopic mechanisms accountable for the uniaxial magnetocrystalline anisotropy, strong enough to overcome the shape anisotropy in films thicker than $20 \mathrm{~nm}$ and reverse the magnetization perpendicularly to the film plane. With the aim to study magnetic properties on the atomic scale, we have undertaken a thorough investigation of a series of $\mathrm{Mn}_{5} \mathrm{Ge}_{3}$ epitaxial films with thicknesses varying between 9 and $300 \mathrm{~nm}$. We have used ${ }^{55} \mathrm{Mn}$ nuclear magnetic resonance (NMR), a powerful local tool giving selective information on manganese ions occupying two crystallographic sites and carrying distinctly different magnetic moments [9]. ${ }^{55} \mathrm{Mn}$ NMR studies in this compound have been previously reported in zero field [10] and in presence of the weak magnetic field (below magnetic saturation) [11]. However, these previous studies have been carried out on the randomly oriented polycrystalline samples and do not help to understand the anisotropic properties. Besides, due to the reported unusual behavior of NMR lines from two Mn crystal sites in presence of magnetic field [11], their shift in opposite directions calls for a closer look at this observation. 


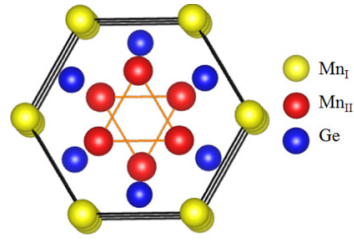

Top view

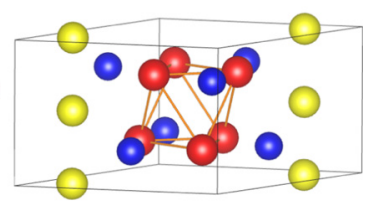

Side view

FIG. 1. Crystal structure for $\mathrm{Mn}_{5} \mathrm{Ge}_{3}$. Left: top view on $x y$ plane, right: side view. Colored spheres denote $\mathrm{Mn}_{\mathrm{I}}$ (yellow), $\mathrm{Mn}_{\mathrm{II}}$ (red), and Ge (blue). This drawing was prepared by means of VESTA software [5].

In this work, we have adopted the following methodology: first we analyze the properties of zero-field ${ }^{55} \mathrm{Mn}$ NMR spectrum in 300-nm film, where the magnetization direction is well defined along the hexagonal $c$ direction, enforced by the strong magnetocrystalline anisotropy. As a next step, we study the evolution of NMR spectrum in the $300-\mathrm{nm}$ film as a function of the external magnetic field applied along the $c$ direction and in the $c$ plane. By increasing the field strength beyond magnetic saturation in the respective crystallographic directions, we obtain important information on the anisotropy of hyperfine fields in the two crystallographic Mn sites. This knowhow will be used to monitor the spontaneous reorientation of magnetic easy direction by following the zero-field NMR spectra recorded from films in the thickness range from 9 to $300 \mathrm{~nm}$.

\section{EXPERIMENTAL DETAILS}

In order to obtain high-quality epitaxial films of $\mathrm{Mn}_{5} \mathrm{Ge}_{3}$, all the samples have been elaborated in an ultrahigh-vacuum molecular beam epitaxy (MBE) setup with a base pressure less than $10^{-10}$ Torr and equipped with high-purity Ge and $\mathrm{Mn}$ evaporation cells. After conventional chemical and thermal cleaning [6], a thick Ge buffer layer was first deposited on a $\mathrm{Ge}(111)$ substrate before the deposition of a Mn layer at room temperature (RT). A subsequent thermal annealing at $600-920 \mathrm{~K}$ is then required to activate interdiffusion and nucleation processes from the interface, leading to the formation of one single phase as demonstrated by the x-ray diffraction data presented in Fig. 2. Only the $\mathrm{Mn}_{5} \mathrm{Ge}_{3}(002)$ and (004) reflections are observed, which correspond to the atomic planes perpendicular to the $c$ axis of the $\mathrm{Mn}_{5} \mathrm{Ge}_{3}$ hexagonal lattice. These results are confirmed by a transmission electron microscope (TEM) analysis (inset of Fig. 2), revealing that the $\mathrm{Mn}_{5} \mathrm{Ge}_{3}$ films are fully epitaxial with the $c$ axis parallel to $\mathrm{Ge}\langle 111\rangle$, that is perpendicular to the interface [7].

Zero-field ${ }^{55} \mathrm{Mn}$ NMR spectra have been recorded at $4.2 \mathrm{~K}$ with an automatic, phase-sensitive spin-echo spectrometer using a broadband NMR probe [12]. NMR signal intensity has been corrected for the frequency variation of the NMR enhancement factor according to the Panissod protocol [13], which involves recording several spectra at different values of the radio-frequency excitation field (rf field) and computing its optimum value as well as the NMR enhancement factor at each frequency. This procedure gives the intrinsic intensity distribution, signal intensity being proportional only to the number of nuclei resonating at a given frequency.

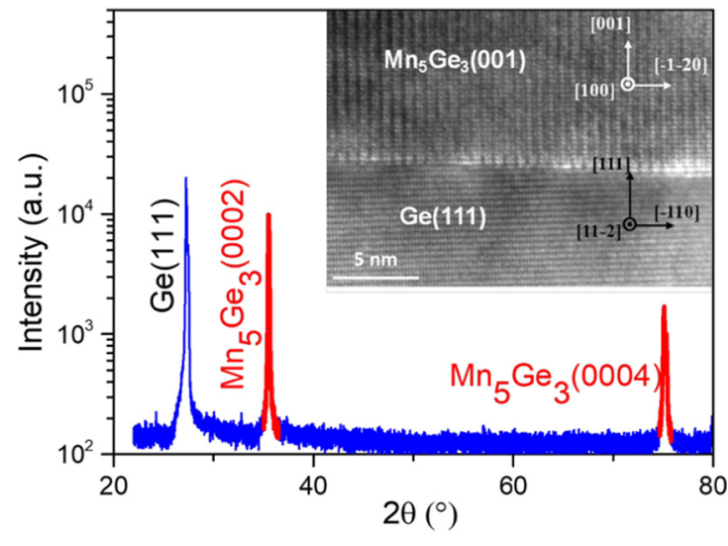

FIG. 2. XRD spectrum of a thin film of $\mathrm{Mn}_{5} \mathrm{Ge}_{3}$ epitaxially grown on $\mathrm{Ge}(111)$ in $\theta-2 \theta$ geometry. Inset: high-resolution crosssectional TEM image, showing the interface of the heterostructure $\mathrm{Mn}_{5} \mathrm{Ge}_{3} / \mathrm{Ge}(111)$.

On the other hand, the experiments in presence of the external magnetic field were carried out at a single value of the rf field amplitude using the NMR probe that is tuned to resonance at each frequency to increase the signal-to-noise ratio [14]. These experiments were aimed at investigation of the local magnetic fields, thus, the only parameter of interest was the frequency of particular NMR lines and not their intrinsic intensity.

\section{RESULTS AND DISCUSSION}

\section{A. 300-nm epitaxial film of $\mathrm{Mn}_{5} \mathrm{Ge}_{3}$, zero external field}

Figure 3 presents the ${ }^{55} \mathrm{Mn}$ NMR spectrum recorded from the $\mathrm{Mn}_{5} \mathrm{Ge}_{3}$ film with thickness of $300 \mathrm{~nm}$. The spectrum consists of two components in distinctly different frequency ranges. The quintuplet centered at frequency $207.5 \mathrm{MHz}$ is readily assigned to the $\mathrm{Mn}$ atoms located in the $4 d$ sites, whereas a structureless $\mathrm{Mn}_{\text {II }}$ line at frequency $428 \mathrm{MHz}$ to the $\mathrm{Mn}$ atoms in $6 g$ sites. The $4 d$ site is characterized by

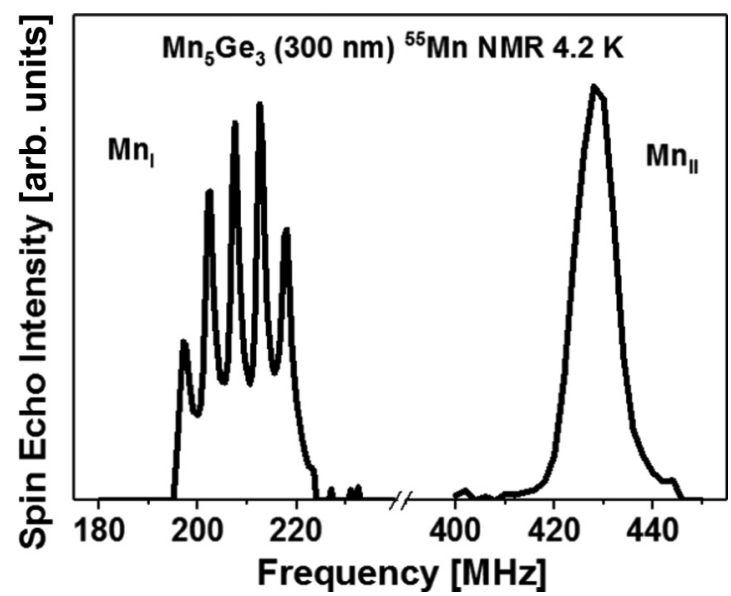

FIG. 3. ${ }^{55} \mathrm{Mn}$ NMR spectrum recorded from the 300-nm-thick $\mathrm{Mn}_{5} \mathrm{Ge}_{3}$ film at $4.2 \mathrm{~K}$ in the absence of external magnetic field. Signal intensity has been corrected for the frequency variation of the NMR enhancement factor using the Panissod protocol [13]. 


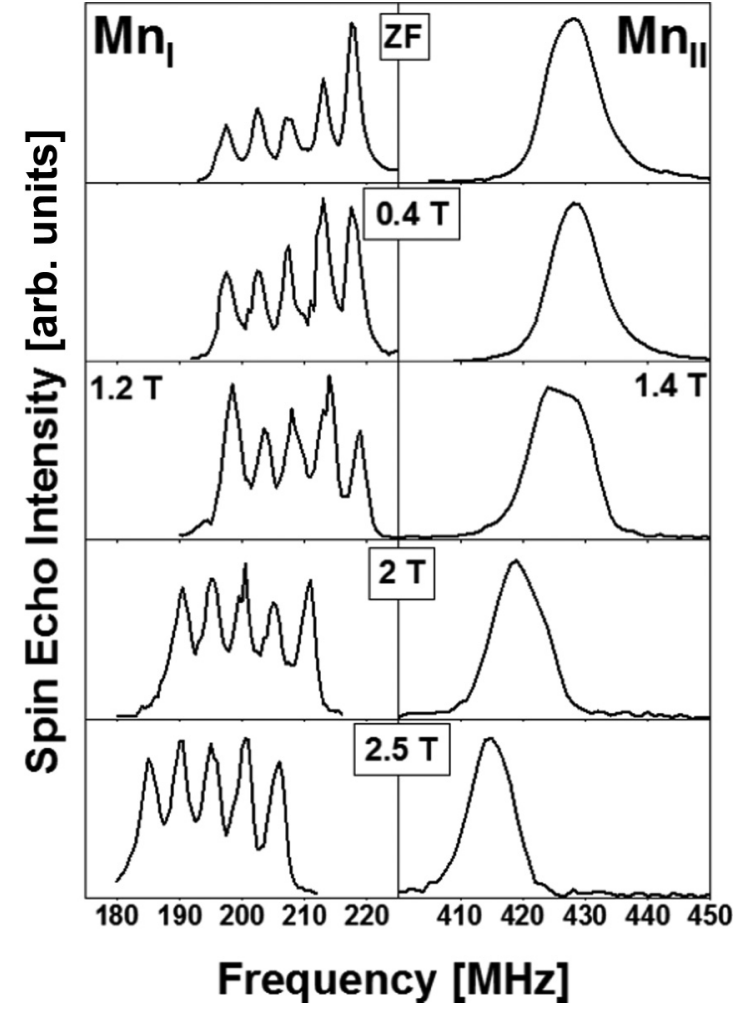

FIG. 4. ${ }^{55} \mathrm{Mn}$ NMR spectra at the $\mathrm{Mn}_{\mathrm{I}}$ site (left panels) and $\mathrm{Mn}_{\text {II }}$ site (right panels) of $\mathrm{Mn}_{5} \mathrm{Ge}_{3}$ recorded from the 300-nm-thick epitaxial film at $4.2 \mathrm{~K}$ at different values of the external magnetic field applied perpendicular to the film plane (i.e., along the crystallographic $c$ axis). The relative intensities of some NMR lines are somewhat different than in case of the NMR spectrum presented in Fig. 3. This is because the frequency dependence of the NMR enhancement factor has not been accounted for, as described in the experimental section.

uniaxial symmetry, giving rise to strong electric field gradient $(E F G)$, which interacts with the quadrupole momentum of the Mn nucleus, resulting in a characteristic structure of the NMR line consisting of five components, as expected in case of nuclear spin $I=\frac{5}{2}$ [15]. The large frequency gap between the two spectrum components is due to a significant difference between the Mn magnetic moments in the two sites: $1.94 \mu_{B}$ at the $4 d$ site and $3.34 \mu_{B}$ at the $6 g$ site [9]. The observed NMR spectrum corresponds closely to the zero-field ${ }^{55} \mathrm{Mn}$ NMR spectrum previously reported in polycrystalline samples of this material [11]. However, further experiments carried out in presence of external magnetic field, as described in the following paragraphs, indicate the need to revisit some aspects of the interpretation given in Ref. [11].

\section{B. 300-nm epitaxial film of $\mathrm{Mn}_{5} \mathrm{Ge}_{3}$, external field perpendicular to film plane}

Figure 4 presents the NMR spectra recorded at a given (arbitrary) amplitude of the rf field for different values of the external dc magnetic field applied in the out-of-plane direction, i.e., along the crystallographic $c$ axis. One readily notes that for the small values of the external magnetic field (below magnetic saturation), the frequency position of all NMR lines does not change. Once the saturation in the hexagonal $c$ direction is

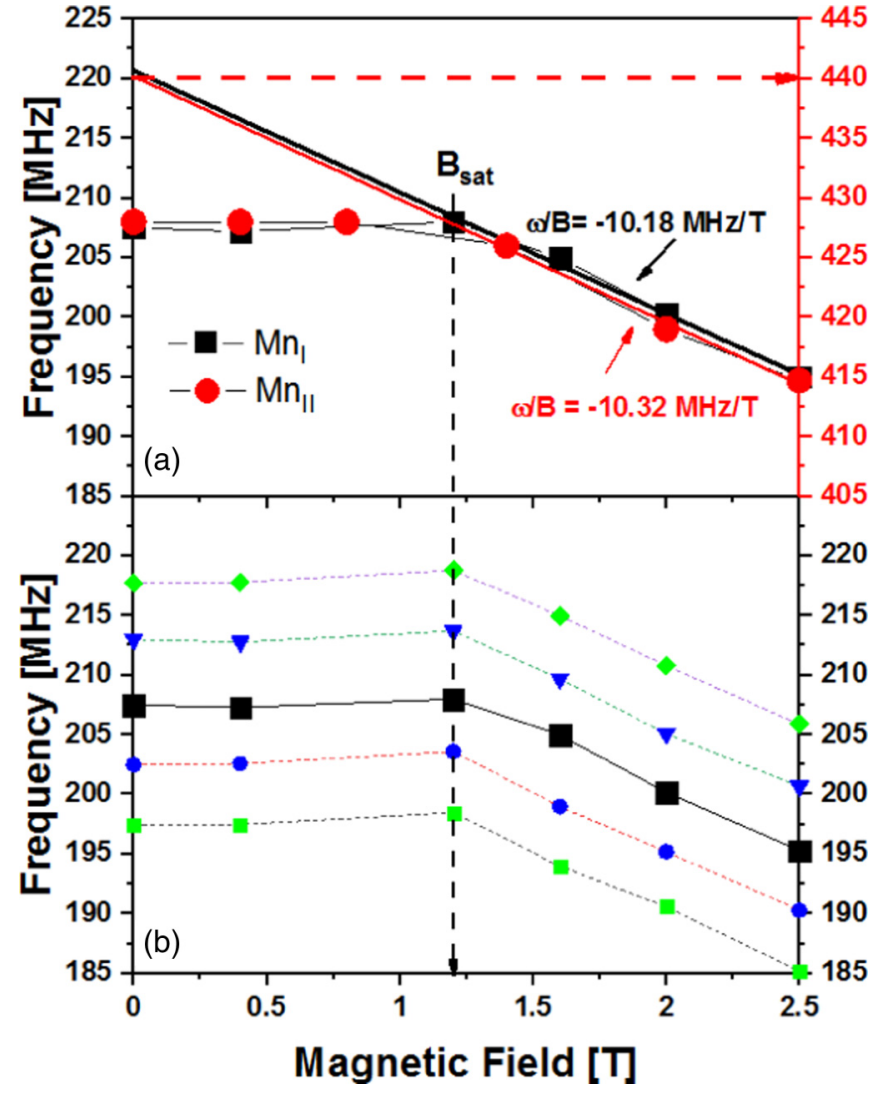

FIG. 5. ${ }^{55} \mathrm{Mn}$ NMR frequency versus magnetic field applied in the $c$ direction of the epitaxial 300-nm film of $\mathrm{Mn}_{5} \mathrm{Ge}_{3}$ : (a) black squares and the left scale: central frequency of the NMR spectrum from $\mathrm{Mn}_{\mathrm{I}}$ site; red circles and the right scale - from the $\mathrm{Mn}_{\mathrm{II}}$ site; (b) central line and quadrupolar satellites of the NMR spectrum from the $\mathrm{Mn}_{\mathrm{I}}$ site.

reached (around 1.2 T), the NMR spectra shift uniformly towards low frequencies. This is better visible in Figs. 5(a) and 5(b), where we plot the NMR frequencies vs external magnetic field for all spectrum components.

For the external field values weaker than that required to magnetically saturate the sample, the effect of magnetic field is shielded by the domain structure. The domains aligned along the field direction are expanding and those oppositely magnetized are shrinking, giving rise to the nonzero magnetization and to the demagnetizing field associated with it. The external field is compensated by the demagnetizing field and the effective local magnetic field acting at the nuclear spins is not altered, therefore, the NMR frequency remains constant in this field range, as seen in Fig. 5.

The general resonance condition, including all contributions to the effective magnetic field at nucleus, is given by

$$
\frac{\vec{\omega}}{\gamma}=\vec{B}_{\mathrm{eff}}=\vec{B}_{\mathrm{ext}}+\vec{B}_{\mathrm{dem}}+\vec{B}_{h f}
$$

where $\vec{\omega}$ is the resonance frequency, $\gamma$ is gyromagnetic ratio, $\vec{B}_{\text {eff }}$ is the effective field at nuclei corresponding to sum of the external field $\vec{B}_{\text {ext }}$, demagnetizing field $\vec{B}_{\text {dem }}$, and hyperfine field $\vec{B}_{h f}$. At magnetic saturation, the demagnetizing field reaches the maximum value. With further increasing the external field strength, both NMR lines shift towards lower 
TABLE I. NMR frequencies and corresponding effective fields on the two Mn sites in $\mathrm{Mn}_{5} \mathrm{Ge}_{3}$ and demagnetizing field values determined from extrapolation of experimental results.

\begin{tabular}{lccccc}
\hline \hline \multirow{2}{*}{ Site } & \multicolumn{2}{c}{ NMR frequency $(\mathrm{MHz})$} & \multicolumn{2}{c}{ Effective field on nucleus (T) } & \multirow{2}{*}{ Demagnetizing field (T) } \\
& Demagnetized sample & Single domain & Demagnetized sample & Single domain & \\
\hline $\mathrm{Mn}_{\mathrm{I}}$ & 207.5 & 220.7 & 19.67 & 20.92 & 1.25 \\
$\mathrm{Mn}_{\mathrm{II}}$ & 428 & 440.2 & 40.57 & 41.73 & 1.16 \\
\hline \hline
\end{tabular}

frequencies. The slope of this decline is $10.18 \mathrm{MHz} / \mathrm{T}$ for the $\mathrm{Mn}_{\mathrm{I}}$ line and $10.32 \mathrm{MHz} / \mathrm{T}$ for the $\mathrm{Mn}_{\mathrm{II}}$ line; these values are very close to the ${ }^{55} \mathrm{Mn}$ gyromagnetic ratio $(10.55 \mathrm{MHz} / \mathrm{T})$. The extrapolation of this linear dependence to zero external field gives the NMR frequency expected in a single-domain material for the center of $\mathrm{Mn}_{\mathrm{I}}$ and $\mathrm{Mn}_{\mathrm{II}}$ line (220.7 and 440.2 $\mathrm{MHz}$, respectively) and make it possible to evaluate the demagnetizing field in the magnetically saturated sample (Table I).

The value of demagnetizing field obtained from the NMR frequency at each $\mathrm{Mn}$ site is around $1.2 \mathrm{~T}$, corresponding indeed to the observed point of flexure of the line describing NMR frequency vs field dependence on Fig. 5. The negative slope of this line beyond magnetic saturation means that the hyperfine field has an opposite orientation with respect to the external field and, consequently, to the electronic magnetization. To determine the component of hyperfine field responsible for its opposite orientation with respect to the electronic magnetization, let us consider different mechanisms contributing to the hyperfine field. It is well established that the hyperfine field consists of three main contributions [16,17]:

$$
\vec{B}_{h f}=\vec{B}_{h f, c f}+\vec{B}_{h f, \text { orb }}+\vec{B}_{h f, \text { dip }},
$$

where $\vec{B}_{h f, c f}=\widehat{A}_{\text {core }} \vec{\mu}_{\text {loc }}^{s}$ is the contact Fermi term, due to a nonzero spin density at the nucleus resulting from the $s-d$ exchange interaction between the electronic spins in the inner closed $s$ shells and spins of the electrons in the open $3 d$ shell. Those $3 d$ electrons give rise to a local spin moment $\vec{\mu}_{\text {loc }}^{s}$. The contact term of hyperfine interaction is isotropic and the respective tensor $\widehat{A}_{\text {core }}$ can be reduced to the hyperfine interaction constant $A_{\text {core }}$ which has a negative sign for $3 d$ transition metals. Therefore, the contribution of the Fermi contact term to the hyperfine field has opposite orientation with respect to the local spin magnetic moment $\vec{\mu}_{\text {loc }}^{s}$

$\vec{B}_{h f \text {, orb }}=\widehat{A}_{\text {orb }} \vec{\mu}_{\text {loc }}^{l}$ is the orbital contribution due to the unquenched orbital moment of $3 d$ electrons. The elements of hyperfine interaction tensor $\widehat{A}_{\text {orb }}$ have the positive sign with respect to the local orbital moment $\vec{\mu}_{\mathrm{loc}}^{l}$.

$\vec{B}_{h f \text {,dip }}=\widehat{A}_{\text {dip }} \vec{\mu}_{\text {loc }}^{s}$ is the dipolar field due to the spin dipole moments $\left(\vec{\mu}_{\text {loc }}^{s}\right)$ of electrons located in the not fully occupied $3 d$ shells. Magnitude and orientation of this field component depend on symmetry of $3 d$ electron orbitals, but in case of metallic systems it is considered to be negligibly small [18].

Consequently, the observed antiparallel direction of hyperfine field with respect to the electronic magnetization indicates that the main contribution to the hyperfine field originates from the Fermi contact term. However, the anisotropic contribution of $3 d$ electrons (the orbital or dipolar component of the hyperfine field) plays also an important role and becomes evident in the experiments with the external magnetic field applied in the hexagonal $c$ plane, as will be shown in the following paragraphs.

We also note in Fig. 5(b) that the frequency distance between quadrupolar satellites remains constant $(\Delta \omega \sim 5 \mathrm{MHz})$ throughout the investigated field range. This observation carries an information about the respective orientation of the magnetization and the electric field gradient (EFG). It is well established (see, e.g., Ref. [15]) that in the case where quadrupolar interaction is much weaker than the Zeeman interaction and assuming that EFG is of uniaxial symmetry, the frequency distance between two adjacent satellite lines corresponding to transitions between energy levels of nuclear spin I: $(m-1) \longleftrightarrow m$ and $-m \longleftrightarrow-(m-1)$, is described by the formula

$$
\Delta \omega=2 \pi \omega_{Q}\left(m-\frac{1}{2}\right)\left(3 \cos ^{2} \theta-1\right),
$$

where $\omega_{Q}=2 \pi \frac{3 e^{2} q Q}{h 2 I(2 I-1)}$ denotes quadrupolar frequency, $m$ denotes the nuclear magnetic quantum number, and $\theta$ is the angle between EFG and local magnetization directions.

The observed invariance of the quadrupolar frequency indicates that the angle between the (EFG) and the direction of internal magnetic field at the nucleus remains constant when going from the demagnetized state to saturation along the $c$ axis. This means that the signal observed in the demagnetized state originates predominately from the Mn atoms with magnetic moments oriented along the $c$ axis, i.e., those located inside the domains or in domain-wall edges.

\section{300-nm epitaxial film of $\mathrm{Mn}_{5} \mathrm{Ge}_{3}$, external field in the film plane}

Figure 6 shows the NMR spectra in $\mathrm{Mn}_{\mathrm{I}}$ and $\mathrm{Mn}_{\mathrm{II}}$ sites of $\mathrm{Mn}_{5} \mathrm{Ge}_{3}$ recorded at different values of the external in-plane magnetic field. This field induces a progressive rotation of electronic magnetization from the hexagonal $c$ axis towards the $c$ plane. The local magnetic field at Mn nuclei follows this rotation, leading to modification of the NMR spectra.

First observation with regard to the $\mathrm{Mn}_{\mathrm{I}}$ quintuplet line is a progressive decrease of the frequency separation between the satellite lines, from $5 \mathrm{MHz}$ for the demagnetized state down to $2.8 \mathrm{MHz}$ for magnetization oriented in $c$ plane (i.e., for the external field values exceeding the magnetic saturation). This effect is readily explained by the variation of satellite line separation as a function of the angle between the direction of the local magnetic field at a nucleus and the main axis of EFG tensor, given by Eq.. (3). For $\theta=0$, i.e., the EFG and magnetization are parallel, the quadrupole splitting $\Delta \omega$ equals to $\Delta \omega=4 \pi \omega_{Q}\left(m-\frac{1}{2}\right)$, whereas in case of $\theta=\frac{\pi}{2}$ the quadrupole splitting is $\Delta \omega=\left|-2 \pi \omega_{Q}\left(m-\frac{1}{2}\right)\right|$. Therefore, 


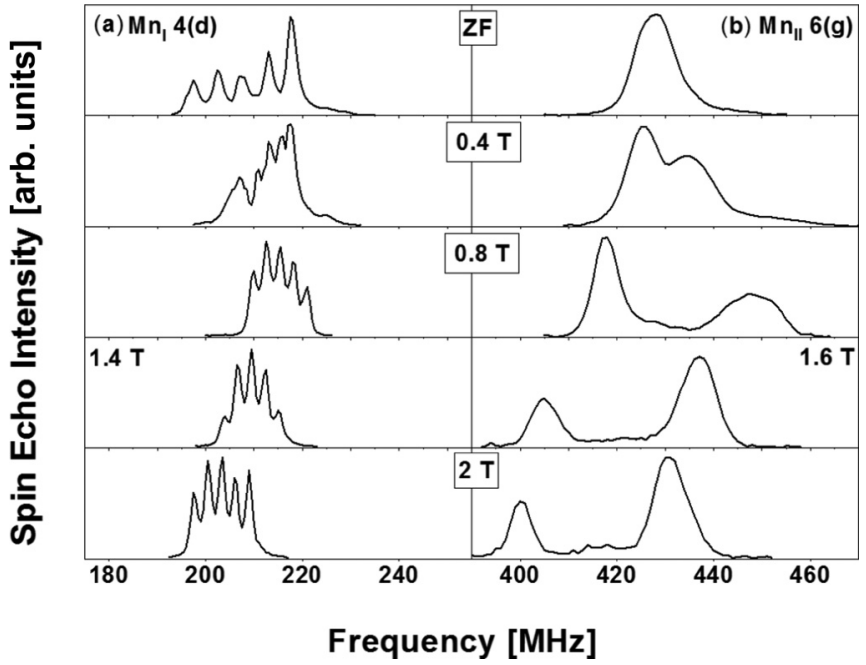

FIG. 6. ${ }^{55} \mathrm{Mn}$ NMR spectra at the $\mathrm{Mn}_{\mathrm{I}}$ site (left panels) and $\mathrm{Mn}_{\text {II }}$ site (right panels) of $\mathrm{Mn}_{5} \mathrm{Ge}_{3}$ recorded from the 300-nm-thick epitaxial film at $4.2 \mathrm{~K}$ at different values of the external magnetic field applied in the film plane (i.e., in the crystallographic $c$ plane).

the observation that the value of $\Delta \omega$ for magnetization oriented along the $c$ axis is twice as large as it is for the $c$ plane means that the $\theta$ angle between the $c$ axis and the local EFG gradient is zero, i.e., the EFG is aligned along the $c$ direction. This is to be expected considering the uniaxial local symmetry of the $\mathrm{Mn}_{\mathrm{I}}$ site, with $c$ axis as the local symmetry axis. Considering that for $\theta=0 \Delta \omega=5 \mathrm{MHz}$, we obtain the quadrupole frequency $\omega_{Q}=0.4 \mathrm{MHz}$ and the corresponding $V_{z z}=e q=5.3 \times 10^{19} \mathrm{~V} / \mathrm{m}^{2}$.

Another effect related to magnetization rotation consists in the peculiar field dependence of the frequency of the ${ }^{55} \mathrm{Mn}$ NMR lines from both crystallographic sites for the in-plane field strength below saturation (Fig. 7). The plot of NMR

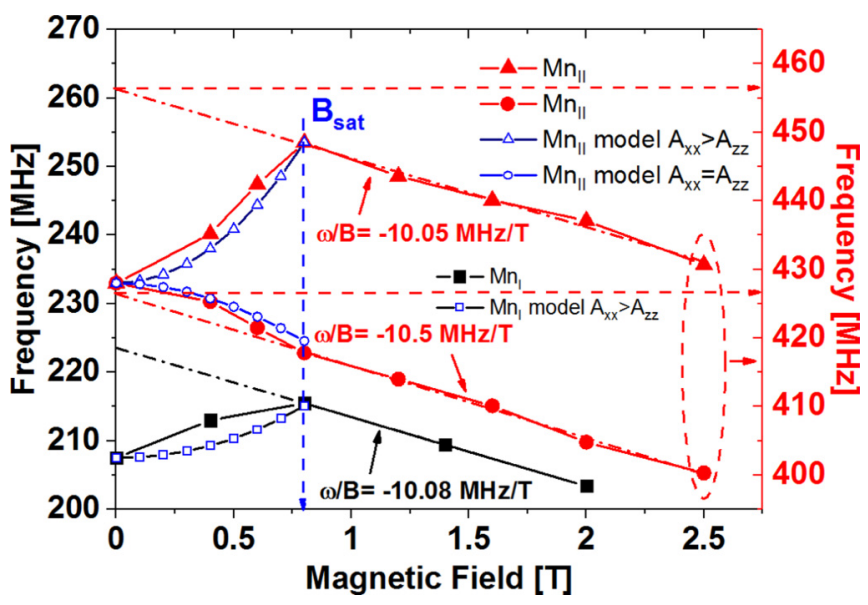

FIG. 7. Frequency of the main ${ }^{55} \mathrm{Mn}$ NMR lines in $\mathrm{Mn}_{5} \mathrm{Ge}_{3}$ film as a function of the in-plane magnetic field strength: (a) left frequency scale and black full squares, central frequency of the NMR spectrum from $\mathrm{Mn}_{\mathrm{I}}$ site; (b) right frequency scale: red full circles and red full triangles, two branches of the NMR line from the $\mathrm{Mn}_{\text {II }}$ site; open blue symbols: the respective branches of the NMR spectrum computed with the use of a model described in Ref. [19]. frequency for the central line of the $\mathrm{Mn}_{\mathrm{I}}$ quintuplet (black squares in Fig. 7), i.e., the line corresponding to $m=\frac{1}{2}$, shows that for the small values of the external field the NMR frequency increases with increasing in-plane field strength. Once the in-plane magnetic saturation is reached, the NMR frequency decreases with a slope of $10.08 \mathrm{MHz} / \mathrm{T}$. The initial increase of NMR frequency accompanying the rotation of magnetization from the $c$ direction towards in-plane orientation is a fingerprint of a strong anisotropy of hyperfine fields between the $c$ direction and the $c$ plane.

Generally, hyperfine field at the nucleus is related to the electronic magnetization [15] by the equation

$$
\vec{B}_{h f}=\widehat{A} \vec{M},
$$

where $\widehat{A}$ is the hyperfine interaction tensor, encompassing contributions from all anisotropic hyperfine field terms listed in Eq. (2). In the local coordinate system, where the $z$ axis is along the hexagonal $c$ direction, this tensor has a form of a diagonal matrix:

$$
\widehat{\mathbf{A}}=\left(\begin{array}{ccc}
A_{x x} & 0 & 0 \\
0 & A_{y y} & 0 \\
0 & 0 & A_{z z}
\end{array}\right) .
$$

The initial increase of the NMR frequency in the $\mathrm{Mn}_{\mathrm{I}}$ site means that the corresponding elements of hyperfine field tensor fulfill the relation $A_{x x}>A_{z z}$, whereas there is no evidence for the in-plane anisotropy on this site, meaning that $A_{x x} \sim A_{y y}$. The in-plane (extrapolated to zero-field) value of the hyperfine field at $\mathrm{Mn}_{\mathrm{I}}$ sites equals to $223.5 \mathrm{MHz} /(10.55 \mathrm{MHz} / \mathrm{T})=$ $21.18 \mathrm{~T}$. The difference between the hyperfine field along $c$ direction and the hyperfine field in $c$ plane $\Delta \vec{B}_{h f}=\left(A_{x x}-\right.$ $\left.A_{z z}\right) \vec{M}$ equals to $1.52 \mathrm{~T}$ and is larger than the in-plane saturation field. This situation is schematically shown in Fig. 8(a). After magnetic saturation in plane is reached, the NMR frequency decreases, as expected, with a slope corresponding to gyromagnetic factor of ${ }^{55} \mathrm{Mn}$ nucleus $(\gamma=10.55 \mathrm{MHz} / \mathrm{T})$.

The spectrum component corresponding to the $\mathrm{Mn}_{\mathrm{II}}$ site reveals even more complex modification upon application of the in-plane magnetic field, as shown in Figs. 6(b) and 7. The NMR line splits in two branches: a new component of the NMR spectrum appears on the high-frequency side of the original line. This means that by tilting the magnetization towards the in-plane orientation, magnetically nonequivalent positions are created among the six $\mathrm{Mn}_{\text {II }}$ sites that are located in two atomic planes within the elementary cell. This is the evidence of a significant in-plane anisotropy of hyperfine fields in this crystallographic position. This situation is schematically represented in Fig. 8(b), showing the top view on all three $\mathrm{Mn}_{\text {II }}$ atoms located in the same atomic plane within the elementary cell. Upon application of the external magnetic field in the film plane, magnetic moments orient themselves towards the field direction, creating two subsets of hyperfine field values. Two $\mathrm{Mn}_{\text {II }}$ atoms having larger $\vec{B}_{h f}$ contribute to high-frequency signal, whereas the third $\mathrm{Mn}_{\text {II }}$ atom [presented here at the top of Fig. 8(b)], characterized by lower hyperfine field value gives rise to the lower-frequency NMR line. Once the in-plane magnetic saturation is reached, defining the orientation of magnetic moments in all $\mathrm{Mn}_{\text {II }}$ atoms, further increase of the external field acts in the opposite direction than the local fields 


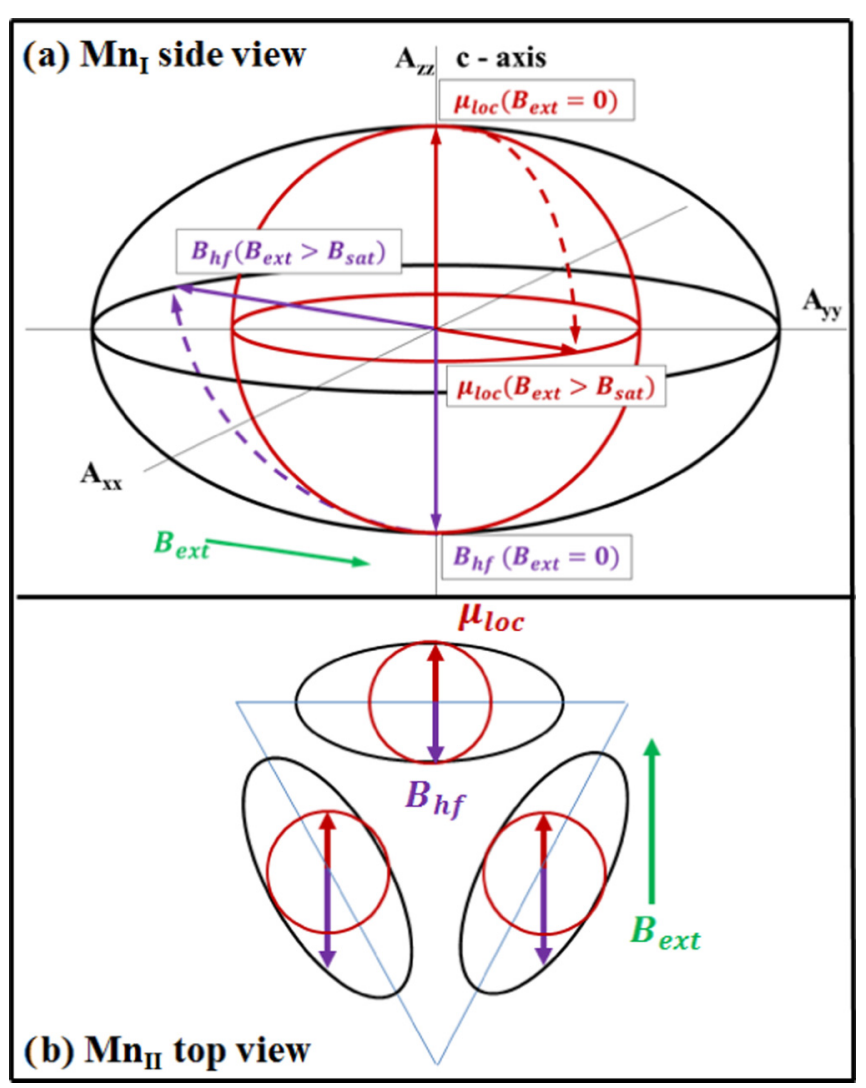

FIG. 8. Schematic representation of local fields distribution in presence of the external magnetic field applied in the $c$ plane: (a) $\mathrm{Mn}_{\text {I }}$ site; (b) $\mathrm{Mn}_{\text {II }}$ site.

at their nuclei and both NMR lines shift uniformly towards lower frequencies.

The anisotropy of hyperfine fields in $\mathrm{Mn}_{\text {II }}$ sites is strongly modulated within the $c$ plane [see Fig. 8(b)]. Choosing the local coordinate system such that the external field is applied along the $x$ axis, for a given $\mathrm{Mn}$ atom (one of the three that are located in the same plane, here the top one) we see that $A_{x x} \neq A_{y y}$, whereas $A_{x x} \sim A_{z z}$, which is evidenced by the uniform decrease of resonance frequency of the lower branch of the NMR signal. By virtue of crystal symmetry, the local (anisotropic) distribution of hyperfine fields in a given $\mathrm{Mn}_{\text {II }}$ site is translated by $60^{\circ}$ rotation to the next neighbor. An analytical model describing this experimental situation leads to the following expression for the NMR frequency as a function of the in-plane external field strength (for details of this model, please see the Supplemental Material [19]):

$$
\begin{aligned}
\omega\left(B_{\text {ext }}\right)= & \omega_{\perp}+\Delta \omega\left(\frac{M B_{\text {ext }}}{2 K}\right)^{2} \\
& -\gamma B_{\text {ext }} \sin \left[\operatorname{arctg}\left(\frac{A_{x x}}{A_{z z}} \sqrt{\frac{\left(\frac{M B_{\text {ext }}}{2 K}\right)^{2}}{1-\left(\frac{M B_{\text {ext }}}{2 K}\right)^{2}}}\right)\right],
\end{aligned}
$$

where $\omega_{\perp}=\gamma A_{z z} \vec{M}$ is the NMR frequnecy for magnetization along the $c$ axis, $\Delta \omega=\gamma \Delta \vec{B}_{h f}=\gamma\left(A_{x x^{\prime}}-A_{z z}\right) \vec{M}$ is the anisotropic contribution due to the difference between hyperfine field along the $c$ direction and in the $c$ plane. Here, $A_{x x^{\prime}}$ denotes the tensor component along an arbitrary direction within $c$ plane.

The third component in this equation describes the projection of the external field $\vec{B}_{\text {ext }}$ on a momentary direction of the hyperfine field. The following values for the parameters have been used: for $\mathrm{Mn}_{\mathrm{I}}, \omega_{\perp}=207.5 \mathrm{MHz}$ and $\Delta \omega=16.09 \mathrm{MHz}$; for $\mathrm{Mn}_{\mathrm{II}}, \omega_{\perp}=428 \mathrm{MHz}$ and for the first branch $\Delta \omega=$ $29.01 \mathrm{MHz}$ whereas for the second branch $\Delta \omega=0$. The field dependence of resonance frequency computed with the use of this model is plotted in Fig. 7 (blue open symbols) and corresponds very closely to the experimental situation.

The experimental values of $\Delta \omega$ correspond to the hyperfine field difference of $1.52 \mathrm{~T}$ between the $c$ axis and the $c$ plane in the $\mathrm{Mn}_{\mathrm{I}}$ site, i.e., the hyperfine field in this site is increased by about $8 \%$ when going from the $c$ axis to the $c$ plane. In case of $\mathrm{Mn}_{\mathrm{II}}$ site this difference oscillates between 0 and $2.77 \mathrm{~T}$ with a period of $60^{\circ}$ due to the sixfold symmetry in the $c$ plane. This corresponds to the increase of hyperfine field value in the $\mathrm{Mn}_{\text {II }}$ site up to $7 \%$ when going from the $c$ axis to the $c$ plane.

The in-plane anisotropy, evidenced by the above experiment and its analysis, calls for the need to revise the results of NMR study presented in Ref. [11]. In case of the in-field experiment, it is now clear that the authors of [11] observed only the lower branch of the NMR spectrum from $\mathrm{Mn}_{\mathrm{II}}$ site, missing the high-frequency branch. Most probably this was due to the polycrystalline character of their samples. The opposite shifts of these two lines in presence of external field were tentatively attributed in Ref. [11] to the transferred hyperfine field component, which would have the opposite sign in two Mn sites. However, our results indicate a different interpretation, pointing clearly to the critical role hyperfine field anisotropy, which splits the $\mathrm{Mn}_{\text {II }}$ line in two branches and determines the in-field behavior of all NMR lines in the magnetically nonsaturated state. The correct interpretation of peculiar features of NMR lines required selective application of the external field along the well-defined crystallographic directions and was only possible due to the good epitaxial quality of our samples.

\section{Magnetization reorientation in very thin films}

Having arrived at the understanding of NMR spectra recorded in the magnetically saturated state, both in plane and out of plane, we can now use them to monitor a spontaneous reorientation of magnetization from the $c$ axis towards $c$ plane in very thin films of $\mathrm{Mn}_{5} \mathrm{Ge}_{3}$, which was previously reported from the analysis of magnetization curves [3] and explained on grounds of magnetostatic considerations [3,7]. The reorientation region was determined to lie between 10 and $20 \mathrm{~nm}$. Figure 9 shows the zero-field NMR spectra recorded from a series of epitaxial films with different thicknesses between 300 and $9 \mathrm{~nm}$. With decreasing film thickness down from $300 \mathrm{~nm}$ (bottom panel), we observe that the NMR lines broaden up and the details of spectrum structure smear out. This can be explained by the progressive evolution of the domain structure in this low- $Q$ material (quality factor defined as $Q=K_{u} / 2 \pi M_{S}^{2}$ was found in the studied materials to be around 0.6 [7]). The fingerprints of NMR signal from regions with in-plane orientation, such as closure domains and domain walls, which were negligible in thicker samples, now gain 


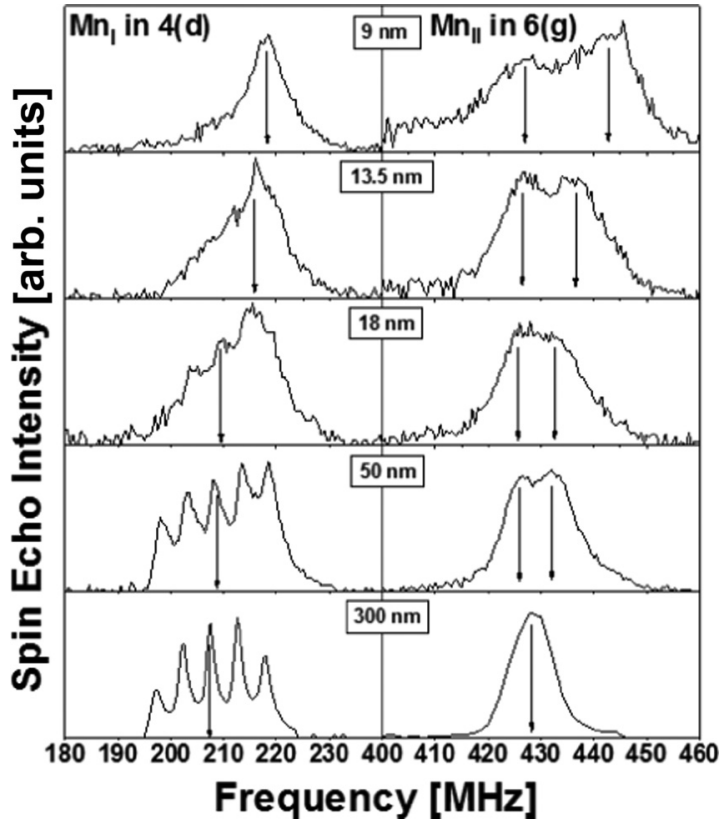

FIG. 9. Zero-field ${ }^{55} \mathrm{Mn}$ NMR spectra recorded at $4.2 \mathrm{~K}$ from the $\mathrm{Mn}_{5} \mathrm{Ge}_{3}$ epitaxial films with different thickness.

in proportion to the signal from domain bulk and contribute to the shape of the NMR spectrum. In the 50-nm film the quadrupolar splitting at the $\mathrm{Mn}_{\mathrm{I}}$ site is still well resolved, but in thinner samples it is eventually lost. Here, the line broadening due to the distribution of orientations in the thin film goes together with the effect of a reduction by a factor of $\frac{1}{2}$ of the quadrupolar splitting as the local field assumes an in-plane orientation. The latter effect is observed and discussed in Fig. 6 where the reorientation was induced by the application of an in-plane field. At the same time, we observe a shift of the central $\mathrm{Mn}_{\mathrm{I}}$ line towards higher frequencies from $207.5 \mathrm{MHz}$ in the 300-nm sample ( $c$-axis orientation) to $218 \mathrm{MHz}$ in the 9-nm film. In case of the $\mathrm{Mn}_{\text {II }}$ site, the new peak appears on the high-frequency side of the original (428-MHz) line and quickly shifts towards higher frequencies as samples get thinner, reaching $444 \mathrm{MHz}$ in the 9-nm film. Clearly, these experiments show that in the lowest thickness limit the NMR spectra reveal the features similar to the NMR spectra recorded from the $300-\mathrm{nm}$ film in the fully saturated state in plane. In samples thinner than $9 \mathrm{~nm}$ the signal-to-noise ratio is very poor, but the peak positions do not change any further, showing that at $9 \mathrm{~nm}$ magnetization is oriented fully in plane. The spin reorientation may simply follow from the shape anisotropy of very thin films, that would be single domain in the perpendicular direction. In thick films, the demagnetization contribution $E_{\mathrm{dem}}$ to the total magnetic energy is suppressed by a multidomain texture. On the other hand, in thin films $E_{\mathrm{dem}}$, significantly lower for the in-plane direction, may overcome the single-ion anisotropy and determine an in-plane reorientation of the moment.

\section{E. Discussion}

We have shown that after reaching magnetic saturation in both the in-plane and out-of-plane directions of epitaxial $\mathrm{Mn}_{5} \mathrm{Ge}_{3}$ films, the NMR frequency displays a linear relation- ship vs magnetic field strength with a negative slope close to the ${ }^{55} \mathrm{Mn}$ gyromagnetic ratio. The only hyperfine field $(h f)$ component providing contribution antiparallel with respect to the magnetic moment is the contact Fermi term, proportional to the spin density at the nucleus [see the discussion of Eq. (2)]. This isotropic $h f$ term represents the main contribution to hyperfine fields on the two Mn sites and accounts for the decrease of resonance frequency in magnetically saturated state. However, the experiments with external magnetic field applied in the film plane reveal an additional presence of another $h f$ component, pointing in the opposite direction, i.e., oriented along the electronic magnetization, and responsible for the initial increase of the NMR frequency. This $h f$ component has anisotropic character and is much stronger in the $c$ direction than in the $c$ plane: the difference amounts to $1.52 \mathrm{~T}$ on $\mathrm{Mn}_{\mathrm{I}}$ and up to $2.77 \mathrm{~T}$ on $\mathrm{Mn}_{\mathrm{II}}$ sites. We attribute this anisotropic term to the unquenched orbital moment $\vec{\mu}_{\text {loc }}^{l}$, in accordance with the published results of first-principles calculations [20], where the inclusion of spin-orbit interaction and the resulting orbital contribution was necessary in addition to the Fermi contact field in order to account for hyperfine field values on both $\mathrm{Mn}_{\mathrm{I}}$ and $\mathrm{Mn}_{\mathrm{II}}$ sites. Our results not only support this conclusion, but also provide quantitative information on the magnitude of anisotropy of the orbital contribution to hyperfine fields in the two orthogonal direction. Presence of the small unquenched orbital moment $\vec{\mu}_{\mathrm{loc}}^{l}$ along the $c$ axis is also the most probable source of the observed uniaxial magnetocrystalline anisotropy in this compound, in agreement with the theoretical work of Bruno [21], who has shown the relationship between the orbital moment and magnetocrystalline anisotropy in metals. It can thus be expected that the observed magnetocrystalline anisotropy of $\mathrm{Mn}_{5} \mathrm{Ge}_{3}$ has its origin in the spin-orbit interaction, while the orbital moment is anisotropic in both $\mathrm{Mn}_{\mathrm{I}}$ and $\mathrm{Mn}_{\text {II }}$ sites. Finally, we want to draw readers attention to the recently published results of comprehensive DFT calculations of energy in $\mathrm{Mn}_{5} \mathrm{Ge}_{3}$, which clearly point out at the anisotropy of orbital moment as a source of magnetic anisotropy [22]. Our result provides a direct experimental observation of the anisotropy of the orbital moment in this system.

\section{CONCLUSIONS}

By performing experiments in external magnetic field strong enough to magnetically saturate the film in the two orthogonal directions, we have demonstrated a significant anisotropy of hyperfine fields on Mn nuclei in the two $\mathrm{Mn}$ crystallographic sites of $\mathrm{Mn}_{5} \mathrm{Ge}_{3}$ structure and determined the magnitude of the anisotropic part of the orbital contribution to hyperfine field. Due to this anisotropic contribution of the hyperfine field the $4 d$ site $\left(\mathrm{Mn}_{\mathrm{I}}\right)$ displays an anisotropy of $8 \%$ between the hexagonal $c$ direction and the $c$ plane. In the $6 g\left(\mathrm{Mn}_{\mathrm{II}}\right)$ site, the strength of hyperfine field anisotropy between the $c$ plane and the $c$ direction is strongly modulated and varies between $0 \%$ and $7 \%$, due to the presence of an additional sixfold anisotropy in the $c$ plane. This study provides experimental insight into the hyperfine fields of $\mathrm{Mn}_{5} \mathrm{Ge}_{3}$, correcting the previously published NMR report [11]. 
[1] R. P. Panguluri, Ch. Zeng, H. H. Weitering, J. M. Sullivan, S. C. Erwin, and B. Nadgorny, Phys. Status Solidi B 242, R67 (2010).

[2] I. Slipukhina, E. Arras, Ph. Mavropoulos, and P. Pochet, Appl. Phys. Lett. 94, 192505 (2009).

[3] A. Spiesser, F. Virot, L. A. Michez, R. Hayn, S. Bertaina, L. Favre, M. Petit, and V. Le Thanh, Phys. Rev. B 86, 035211 (2012).

[4] E. Parthe and W. Jeitschko, Acta Crystallogr. 19, 1031 (1965).

[5] K. M. nad F. Izumi, J. Appl. Crystallogr. 44, 1272 (2011).

[6] S. Olive-Mendez, A. Spiesser, L. A. Michez, V. Le Thanh, A. Glachant, J. Derrien, T. Devillers, A. Barski, and M. Jamet, Thin Solid Films 517, 191 (2008).

[7] L. A. Michez, A. Spiesser, M. Petit, S. Bertaina, J. F. Jacquot, D. Dufeu, C. Coudreau, M. Jamet, and V. Le Thanh, J. Phys.: Condens. Matter 27, 266001 (2015).

[8] R. Kalvig, E. Jedryka, P. Aleshkevych, M. Wojcik, W. Bednarski, M. Petit, and L. Michez, J. Phys. D: Appl. Phys. 50, 125001 (2017).

[9] J. B. Forsyth and P. J. Brown, J. Phys.: Condens. Matter 2, 2713 (1990).

[10] R. F. Jackson, R. G. Scurlock, D. B. Utton, and E. M. Wray, Proc. Phys. Soc., London 85, 127 (1965).
[11] P. Panissod, A. Quachaou, and G. Kappel, J. Phys. C: Solid State Phys. 17, 5799 (1984).

[12] S. Nadolski, M. Wojcik, E. Jedryka, and K. Nesteruk, J. Magn. Magn. Mater. 140-144, 2187 (1995).

[13] P. Panissod, M. Malinowska, E. Jedryka, M. Wojcik, S. Nadolski, M. Knobel, and J. E. Schmidt, Phys. Rev. B 63, 014408 (2000).

[14] G. Allodi, A. Banderini, R. D. Renzi, and C. Vignali, Rev. Sci. Instrum. 76, 083911 (2005).

[15] A. Abragam, Principles of Nuclear Magnetism (Oxford University Press, Oxford, 1961).

[16] R. E. Watson and A. J. Freeman, Phys. Rev. 123, 2027 (1961).

[17] A. Narath, Hyperfine Interactions (Academic, New York, 1967).

[18] W. Marshall and C. E. Johnson, J. Phys. Radium 23, 733 (1962).

[19] See Supplemental Material at http://link.aps.org/supplemental/ 10.1103/PhysRevB.97.174428 for NMR frequency as a function of the in-plane external field strength.

[20] S. Picozzi, A. Continenza, and A. J. Freeman, Phys. Rev. B 70, 235205 (2004).

[21] P. Bruno, Phys. Rev. B 39, 865 (1989).

[22] H. K. Yuan, H. Cui, B. Liu, C. L. Tian, J. Z. Wang, A. L. Kuang, and H. Chen, J. Chem. Phys. 148, 074701 (2018). 